\title{
Supporting information for In Situ Small-angle X-ray Scattering Studies on the Growth Mechanism of Anisotropic Platinum Nanoparticles
}

Wataru Yoshimune*, Akira Kuwaki*, Takumi Kusano, Takuro Matsunaga, and Hiroshi Nakamura

Toyota Central R\&D Labs, Inc., 41-1 Yokomichi, Nagakute, Aichi 480-1192, Japan 


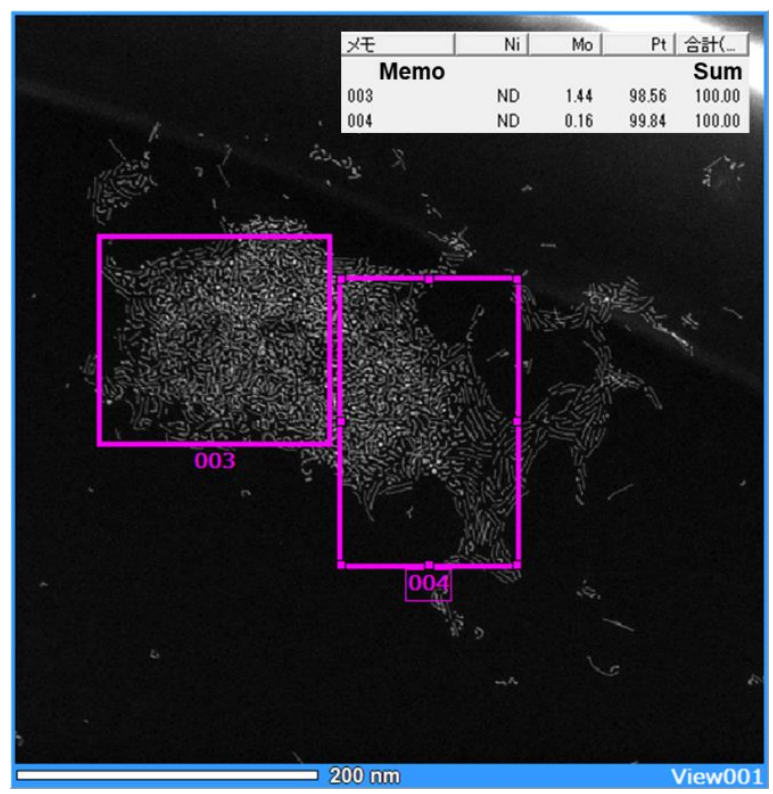

Figure S1. Results of energy-dispersive X-ray spectroscopy (EDS) analysis: platinum nanoparticles (NPs) synthesized by heating at Sequence $3\left(T_{\mathrm{R}}=100^{\circ} \mathrm{C}, t_{\mathrm{R}}=75 \mathrm{~min}\right)$. The EDS analysis was examined by JEOL JEM-2100F.

(a) Sequence 1

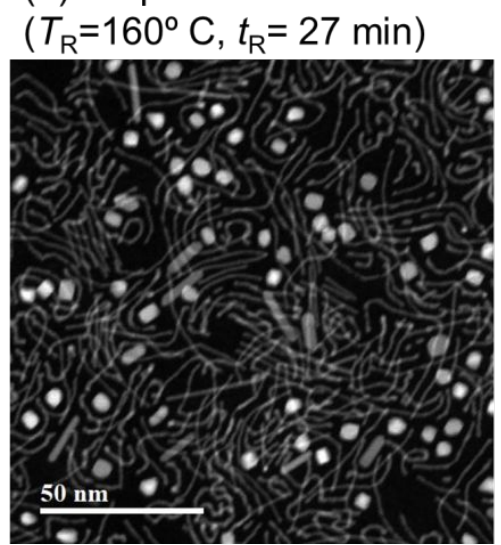

(b) Sequence 3

$$
\left(T_{\mathrm{R}}=100^{\circ} \mathrm{C}, t_{\mathrm{R}}=15 \mathrm{~min}\right) \quad\left(T_{\mathrm{R}}=100^{\circ} \mathrm{C}, t_{\mathrm{R}}=75 \mathrm{~min}\right)
$$

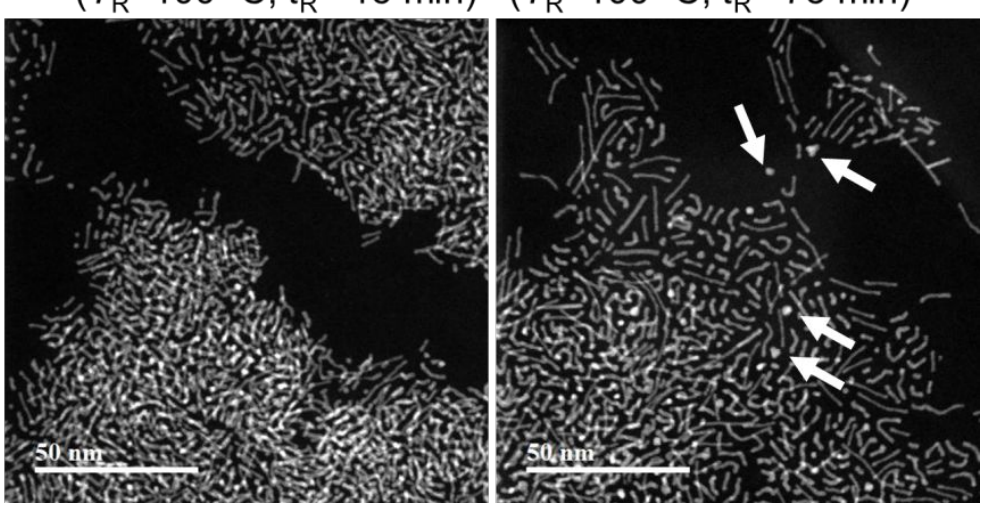

Figure S2. Transmission electron microscope (TEM) images of synthesized platinum NPs sampled at different reaction conditions: (a) Sequence $1\left(T_{\mathrm{R}}=160^{\circ} \mathrm{C}, t_{\mathrm{R}}=27 \mathrm{~min}\right)$, (b) Sequence $3\left(T_{\mathrm{R}}=100^{\circ} \mathrm{C}, t_{\mathrm{R}}=15 \mathrm{~min}\right)$, and (c) Sequence $3\left(T_{\mathrm{R}}=100^{\circ} \mathrm{C}, t_{\mathrm{R}}=75 \mathrm{~min}\right)$. Arrows in (c) indicate low aspect particles.

(a) Sequence 1

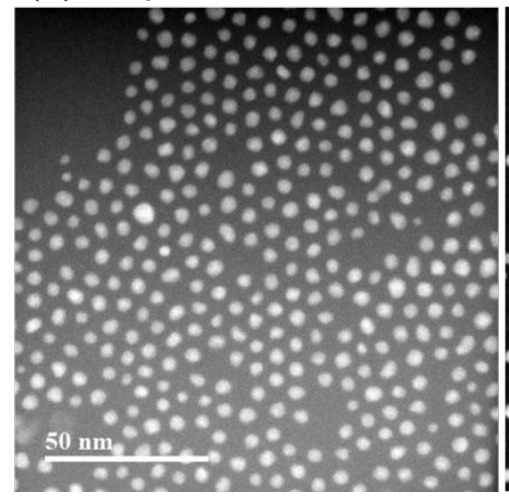

(b) Sequence 2

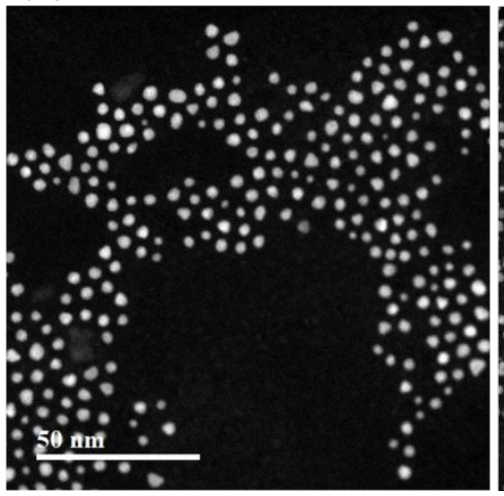

(c) Sequence 3

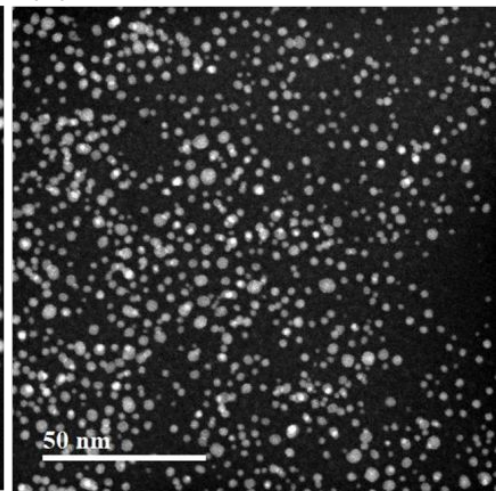


Figure S3. TEM images of synthesized platinum NPs at small-angle X-ray scattering (SAXS) measurements: (a) Sequence 1, (b) Sequence 2, and (c) Sequence 3.

(a) Sequence 1

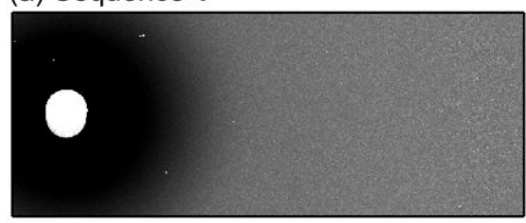

(b) Sequence 2

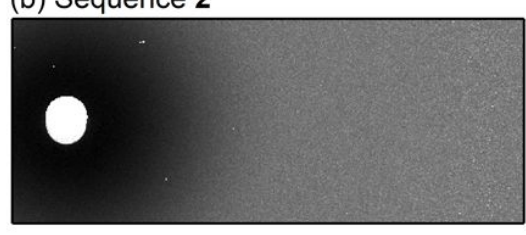

(c) Sequence 3

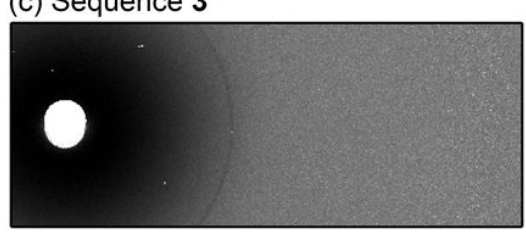

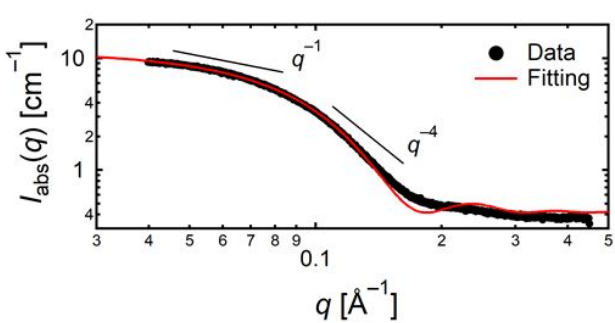
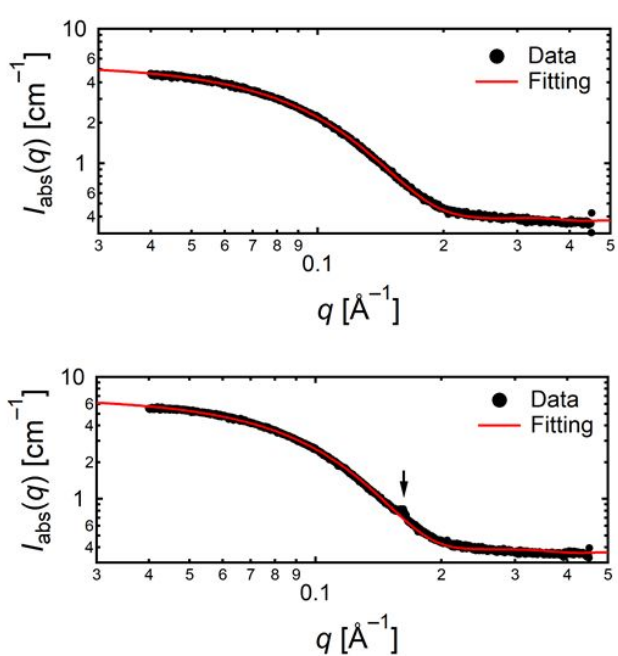

Figure S4. (left) 2D SAXS patterns and (light) circular averaged 1D SAXS profiles for anisotropic platinum NPs: (a) Sequence 1, (b) Sequence 2, and (c) Sequence 3.
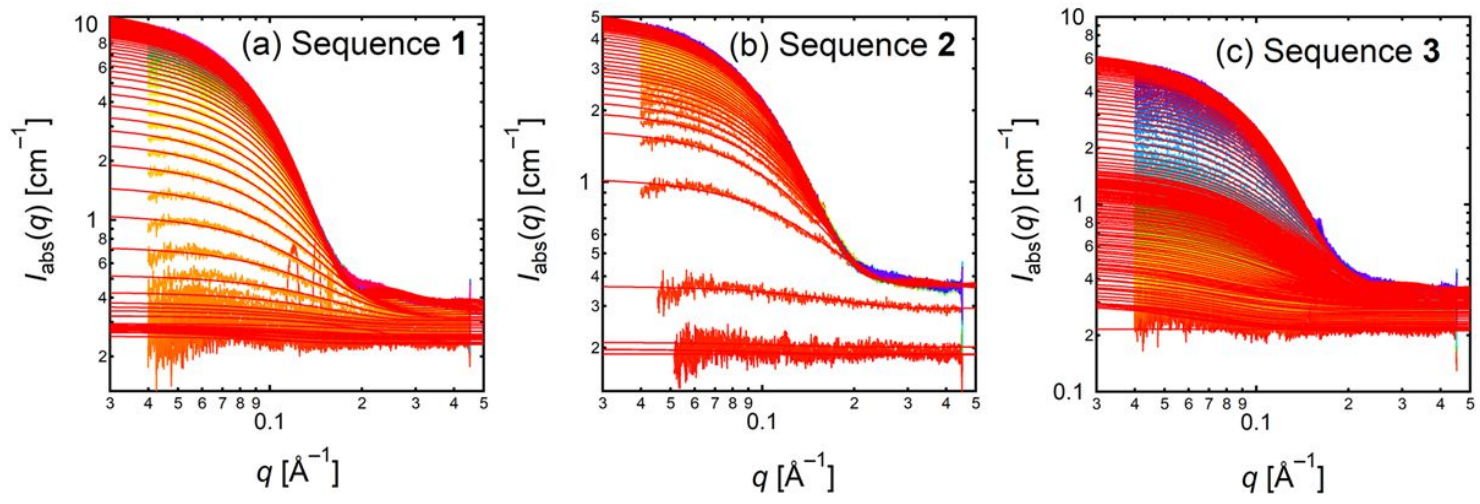

Figure S5. All fitting curves of in situ time-sliced SAXS profiles: (a) Sequence 1, (b) Sequence 2, and (c) Sequence 3. 


\section{Sequence 3}

$\left(T_{\mathrm{R}}=100^{\circ} \mathrm{C}, t_{\mathrm{R}}=75 \mathrm{~min}\right)$

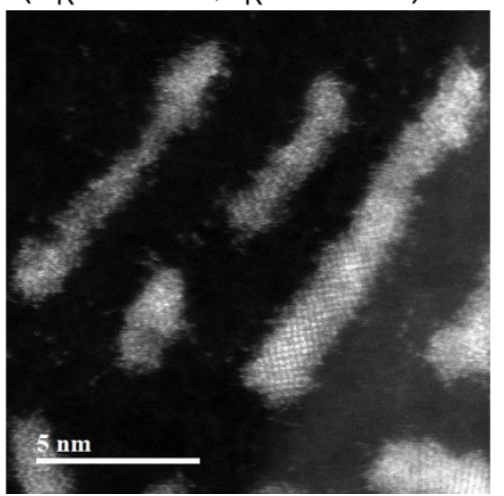

Figure S6. High-resolution TEM images of synthesized platinum NPs sampled at Sequence $3\left(T_{\mathrm{R}}=100^{\circ} \mathrm{C}, t_{\mathrm{R}}=75 \mathrm{~min}\right)$. 